\title{
Untargeted Metabolomics Analysis of Serum Metabolites in Zucker Diabetic Fatty Rats
}

\author{
Qingyuan Liu ${ }^{*}$, and Sze Ying Quach ${ }^{2}$ \\ ${ }^{1}$ Trafalgar Castle Schools, 401 Reynolds Street, Whitby, ON, Canada \\ ${ }^{2}$ Trafalgar Castle Schools, 401 Reynolds Street, Whitby, ON, Canada
}

\begin{abstract}
Zucker Diabtic Fatty rats is a common model used for the studies related to type 2 diabetes. Metabolomics, proven by previous studies, can be used for the prediction of phenotype. This investigation held untargeted metabolomics analysis on the serum metabolites of the Zucker Diabetic Fatty rats, intended to gain insights in the study of the pathology of type 2 diabetes. LCMS method (liquid chromatography coupled with mass spectrometry) is an analytical chemistry technique used to separate and purify complex mixtures. After identifying metabolites using MS2 library spectrum matching, this investigation used Metabo Analyst to compare fatty rats and lean rats. Lastly, by applying over representation analysis, we identified seven metabolic pathways that are important for humans, which assists in discovery of therapeutic targets and the developments of diabetes biomarkers.
\end{abstract}

\section{Introduction}

\subsection{Background}

The Zucker Diabetic Fatty rat is a labotory model [1] commonly used in the studies associated with type 2 diabetes, due to its characters of ravenous, obese, hyperlipidemic, hyperinsulinemic, and hyperglycemic, that are typical traits of the human disease [2]. Metabolomics is the general description of metabolites in biological systems, including products of metabolism that are used for energy storage and utilization, precursors of proteins and carbohydrates, and et cetera [3]. Studies have shown that metabolomics serves for the prediction of the biological phenotype [4], therefore, the analysis of serum metabolites in Zucker Diabetic Fatty rats would be used as an investigation pathway to the pathology of type 2 diabetes.

\subsubsection{Zucker Diabetic Fatty Rats}

Zucker Diabetic Fatty rats have always been used in studies of type 2 diabetes related to obesity [5]. Their age range of diabetes onset is between ten weeks and twenty weeks [5]. This strain reproduces effectively and therefore is a common model in the disease pathophysiology, therapeutic interventions, and complications study of type 2 diabetes onset of young and middle-aged adults, giving examples like its effects on cardiac metabolism and function $[2,6]$. Therefore, to analyze the underlying metabolic processes of Zucker Diabetic Fatty rats is a way to gain knowledge on the metabolic process in patients with type 2 diabetes onset.

\subsubsection{Metabolomics}

Metabolomics act as the interface between phenotype and genotype. The full understanding of metabolism and other cellular processes brought about structured repositories that can be mathematically expressed [7]. This mathematical expression offers a mechanistic representation of the relation between microbial metabolic phenotype and genotype [7]. Apart from this, genome-scale metabolic models were proven to be valuable for the prediction of organism phenotypes [4], which makes it reasonable for metabolites to reveal aberrant biological processes that are otherwise hard to be observed or confirmed. Therefore, the investigation in the metabolomics of Zucker Diabetic Fatty rats may reveal the unnoticeable causes, mechanisms, and developmental rules of type 2 diabetes.

\subsubsection{Liquid chromatography-Mass Spectrometry (LCMS)}

Mass spectrometry coupled with liquid chromatography (LCMS) is the technique we utilize in the untargeted metabolomics analysis of Zucker Diabetic Fatty rats. It is a popular platform in metabolomic studies and a highthroughput ideal for the analysis of complex mixtures because of its soft ionization and high coverage of metabolites [8]. In addition to this, LCMS separates metabolites and other components from complex compounds based on their retention time. For this reason, the use of this technique allows for quantitative and qualitative analysis of serum metabolites in Zucker

\footnotetext{
*aliceliu0904@gmail.com
} 
Diabetic Fatty Rats, which is an essential part of our investigation [9].

With the rapid advancements in technology, metabolomics starts to be applied in the prediction of incident diabetes and the therapeutic target of type 2 diabetes, such as AMP-activated protein kinase and fructose-6-phosphate amidotransferase $[10,11,12]$. This paper investigates the pathology of type 2 diabetes through the untargeted metabolomic analysis of serum metabolites in Zucker Diabetic Fatty rats, trying to provide some reference and fresh ideas for the invention of new drugs.

\section{Methods}

\subsection{LC-MS configuration for untargeted metabolites}

\subsubsection{Reverse phase liquid chromatography}

We extracted serum metabolites from three fatty rats and three lean rats LC method (reverse-phase liquid chromatography) is used to separate and purify metabolites extracted from the serum of six ZDF rats (three lean rats and three fatty rats) into individual components based on the hydrophobic stationary phase. Reverse-phase liquid chromatography has a high resolving capability which ranges from 2 to 200 and can be applied to a wide range of molecules (Grebe \& Singh, 2011).

\subsubsection{High-resolution orbitrap mass spectrometry}

Mass spectrometry measures the mass-to-charge ratio of ions that can detect qualitative (structure) and quantitative (molecular weight) information. This experiment used a high-resolution orbitrap mass spectrometer with an orbitrap analyzer which is the core component of a mass spectrometer. This type of mass spectrometer will provide exact information. Before When analyte molecules separation has completed in liquid chromatography, molecules would enter mass spectrometry through ESI (electrospray ionization), where molecules will be ionized since mass spectrometry can only detect ions. Ionization was carried out with the following parameters: $4 \mathrm{kV}$ spray voltage, $350{ }^{\circ} \mathrm{C}$ capillary temperature, 45 sheath gas, 8 auxiliary gas, 100 max spray current, 425 probe heat temperature and 55 S-Lens RF Level. The Mass spectrometry ran for $15 \mathrm{~min}$ in a positive mode with 70000 resolutions, $100 \mathrm{~ms}$ maximum IT and 67 to $1000 \mathrm{~m} / \mathrm{z}$ scan range. One thing important is to collect background noise. We need to run mass spectrometry without analytes before running the instrument with analytes, so background noise can be collected by subtracting data of MS without analytes by MS with analytes. To have comprehensive coverage of ions, it's better to scan under both positive and negative modes.

\subsection{Metabolite identification}

Metabolite identification is based on MS2 library spectrum matching. After LC-MS raw files were processed by Compound Discovery 3.1, one ion was isolated and was fragmented by adjusting normalized collision energy. If the fragment of ions matched with the fragment of identified metabolites according to the fragment search database, like $\mathrm{m} / \mathrm{z}$ cloud or human metabolome database (HMDB), the ion could be identified.

\subsection{Comparison between fatty rats and lean rats}

After metabolites were identified, a comparison between fatty rats and lean rats was made to find the significantly altered metabolites by analyzing statistically in MetaboAnalyst. The metabolites were analyzed based on t-test, volcano plot, fold change and heat map. Volcano plot is a type of scatter plot used to identify statistical significance (p-value) and fold change ( $\mathrm{Li}, 2012)$. The ttest is used to determine the significant difference between the two groups. It is also used for the purposes of hypothesis testing in statistics (Kenton, n.d.). Heatmap is a data visualization tool showing people which part gets the most attention.

\subsection{Identification of metabolic pathways}

There are two ways to identify metabolic pathways that both share the principle of over-representation analysis. The first one is to apply identified metabolites using Ingenuity Pathway analysis: mapping identified metabolites onto metabolic pathways and calculate the probability of that happening by random chance. Since there were still a lot of spectral features not identified, we also used the mummichog algorithm to leverage the power of known metabolic pathways to gain functional insight directly from $\mathrm{m} / \mathrm{z}$ features.

\section{Results and Discussion}

\subsection{1 of 2618 spectral features were found significantly altered}

The LCMS separates metabolites and other components from complex compounds based on their retention time. Through the LCMS analysis of the serum of Zucker Diabetic Fatty rats, 2618 spectral features were detected in total. The hierarchical clustering analysis distinguishes the lean group and fatty group clearly (figure 1). There is an obvious change of the metabolic profile from the lean group to the fatty group of Zucker Diabetic Fatty rats. Statistical analysis was conducted on these 2618 spectral features. Spectral features with fold change $>2$, adjusted p-value $(\mathrm{FDR})<0.05$ were determined as significantly altered. However, to be less stringent for mummichog analysis and to obtain more pathway hits, we set the fold change threshold as 1.5 and filtered out spectral features with FDR $>0.1$. Therefore, without the repeated features, 901 significantly altered 
spectral features were found through the statistical analysis (figure 2). Table 1 shows the top 50 significantly altered spectral features, the corresponding fold change and FDR identified by the volcano plot. Two different over-representation analyses were performed on these features.

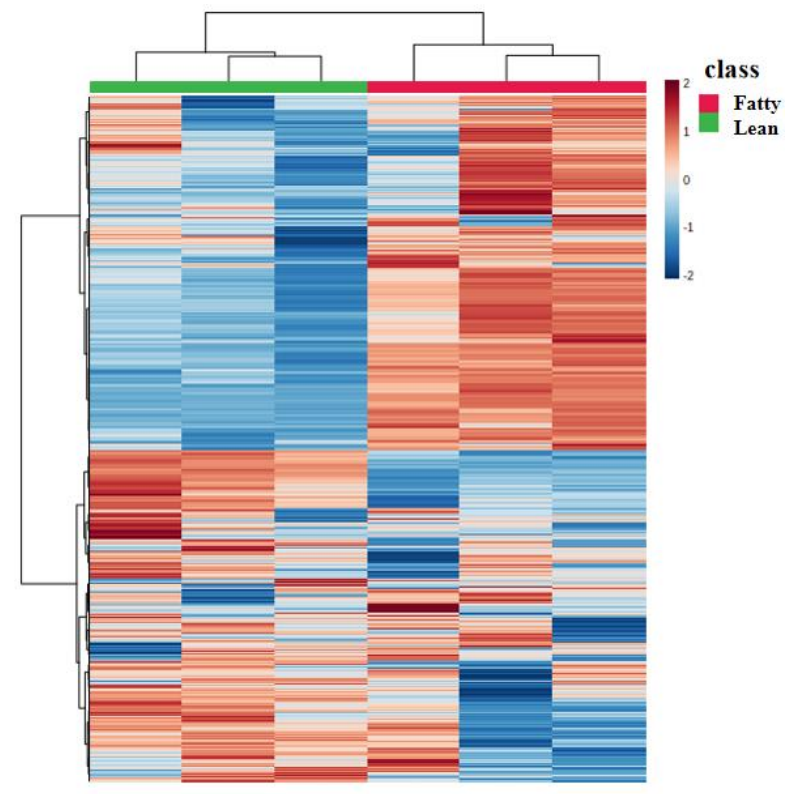

Fatty_3 Fatty_1 Lean_2 Fatty_1 Fatty_2 Fatty_3

Fig. 1. Hierarchical clustering of all samples using all spectral features using Ward algorithm.

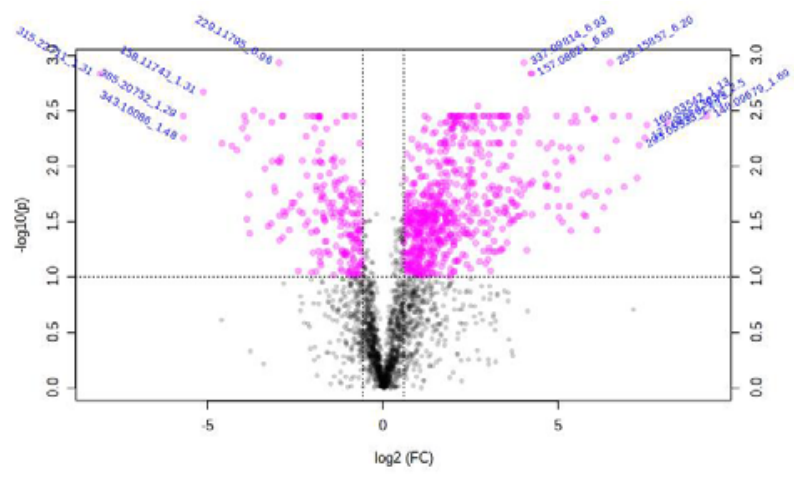

Fig. 2. Volcano plot of spectral features with fold change $>1.5$, $\mathrm{FDR}<0.1$
Table 1. Fold change and corresponding FDR of the top 50 significantly altered spectral features. Spectral features are labeled as ( $\mathrm{m} / \mathrm{z}$-value_retetion time).

\begin{tabular}{|c|c|c|c|c|c|}
\hline & Compounds & FC & $\log 2(\mathrm{FC})$ & p.ajusted & $-\log 10(p)$ \\
\hline 1 & $255.15857 \_6.201$ & 89.03 & 6.4762 & 0.001163 & 2.9344 \\
\hline 2 & $337.09814 \_6.933$ & 16.152 & 4.0136 & 0.001163 & 2.9344 \\
\hline 3 & 229.11795_0.961 & 0.12755 & -2.9709 & 0.001163 & 2.9344 \\
\hline 4 & 315.22711_1.31 & 0.0037093 & -8.0746 & 0.0014669 & 2.8336 \\
\hline 5 & 217.1431_6.932 & 18.928 & 4.2424 & 0.0014669 & 2.8336 \\
\hline 6 & 157.08621_6.692 & 18.55 & 4.2134 & 0.0014669 & 2.8336 \\
\hline 7 & $158.11743-1.317$ & 0.028513 & -5.1323 & 0.0021536 & 2.6668 \\
\hline 8 & $301.14874 \_6.732$ & 6.5015 & 2.7008 & 0.0028814 & 2.5404 \\
\hline 9 & 213.1095_4.578 & 56.619 & 5.8232 & 0.0031195 & 2.5059 \\
\hline 10 & $261.16928 \_6.85$ & 16.684 & 4.0604 & 0.0031195 & 2.5059 \\
\hline 11 & 418.15601_0.983 & 0.077365 & -3.6922 & 0.0031742 & 2.4984 \\
\hline 12 & 148.09679_1.694 & 601.27 & 9.2319 & 0.0035444 & 2.4505 \\
\hline 13 & $531.24695 \_6.726$ & 128.99 & 7.0111 & 0.0035444 & 2.4505 \\
\hline 14 & $555.21686 \_6.726$ & 94.65 & 6.5645 & 0.0035444 & 2.4505 \\
\hline 15 & $385.20752 \_1.292$ & 0.019222 & -5.7011 & 0.0035444 & 2.4505 \\
\hline 16 & 239.08878_3.832 & 44.584 & 5.4785 & 0.0035444 & 2.4505 \\
\hline 17 & $252.11168 \_6.113$ & 30.864 & 4.9478 & 0.0035444 & 2.4505 \\
\hline 18 & $295.15155 \_6.426$ & 17.215 & 4.1056 & 0.0035444 & 2.4505 \\
\hline 19 & $348.12222 \_2.785$ & 0.064242 & -3.9603 & 0.0035444 & 2.4505 \\
\hline 20 & $247.15359-6.693$ & 11.793 & 3.5599 & 0.0035444 & 2.4505 \\
\hline 21 & 133.04945_6.598 & 11.65 & 3.5423 & 0.0035444 & 2.4505 \\
\hline 22 & $203.12767 \_6.733$ & 11.261 & 3.4932 & 0.0035444 & 2.4505 \\
\hline 23 & $160.13304 \_1.187$ & 0.091157 & -3.4555 & 0.0035444 & 2.4505 \\
\hline 24 & $129.0545-6.599$ & 10.56 & 3.4006 & 0.0035444 & 2.4505 \\
\hline 25 & 269.13593_6.691 & 10.455 & 3.3862 & 0.0035444 & 2.4505 \\
\hline 26 & $341.09341 \_6.693$ & 10.402 & 3.3788 & 0.0035444 & 2.4505 \\
\hline 27 & $309.03094 \_6.598$ & 10.044 & 3.3283 & 0.0035444 & 2.4505 \\
\hline 28 & $187.09645 \_6.199$ & 9.3026 & 3.2176 & 0.0035444 & 2.4505 \\
\hline 29 & $247.15361 \_6.73$ & 8.7313 & 3.1262 & 0.0035444 & 2.4505 \\
\hline 30 & $253.08156 \_1.591$ & 8.0375 & 3.0068 & 0.0035444 & 2.4505 \\
\hline 31 & 251.10568_2.011 & 0.13584 & -2.88 & 0.0035444 & 2.4505 \\
\hline 32 & $245.1494 \_2.437$ & 0.13766 & -2.8608 & 0.0035444 & 2.4505 \\
\hline 33 & $161.0808 \_6.118$ & 7.2426 & 2.8565 & 0.0035444 & 2.4505 \\
\hline 34 & $291.17981 \_6.201$ & 7.2301 & 2.854 & 0.0035444 & 2.4505 \\
\hline 35 & $291.11765 \_6.728$ & 6.2029 & 2.633 & 0.0035444 & 2.4505 \\
\hline 36 & 269.13593_6.728 & 6.0158 & 2.5888 & 0.0035444 & 2.4505 \\
\hline 37 & 231.01756_2.1 & 5.7403 & 2.5211 & 0.0035444 & 2.4505 \\
\hline 38 & $227.12756-4.875$ & 5.579 & 2.48 & 0.0035444 & 2.4505 \\
\hline 39 & $289.09546 \_6.112$ & 4.9834 & 2.3171 & 0.0035444 & 2.4505 \\
\hline 40 & $129.05452 \_6.117$ & 4.9568 & 2.3094 & 0.0035444 & 2.4505 \\
\hline 41 & $319.06989 \_4.197$ & 4.6658 & 2.2221 & 0.0035444 & 2.4505 \\
\hline 42 & 233.13809_6.114 & 4.5292 & 2.1793 & 0.0035444 & 2.4505 \\
\hline 43 & $343.04462 \_2.145$ & 0.22134 & -2.1757 & 0.0035444 & 2.4505 \\
\hline 44 & 147.06514 .6 .117 & 4.3383 & 2.1171 & 0.0035444 & 2.4505 \\
\hline 45 & 327.07776_6.114 & 4.3322 & 2.1151 & 0.0035444 & 2.4505 \\
\hline 46 & $231.07388 \_2.353$ & 0.24698 & -2.0175 & 0.0035444 & 2.4505 \\
\hline 47 & $271.08493 \_6.112$ & 3.9838 & 1.9941 & 0.0035444 & 2.4505 \\
\hline 48 & $175.09645 \_6.117$ & 3.9724 & 1.99 & 0.0035444 & 2.4505 \\
\hline 49 & $289.05942 \_4.79$ & 3.8153 & 1.9318 & 0.0035444 & 2.4505 \\
\hline 50 & 317.09039_6.116 & 3.8074 & 1.9288 & 0.0035444 & 2.4505 \\
\hline
\end{tabular}

\subsection{Dysregulated pathways found through ingenuity pathway analysis}

The Ingenuity Pathway Analysis evaluated the overrepresentation of metabolic pathways and identified important pathways affected by the phenotype of type 2 diabetes using identified metabolites. Through the spectrum matching of the MS/MS spectrum of 901 significantly altered features, 44 metabolites were identified and used for Ingenuity Pathway Analysis (figure 3). Using the HMDB of these metabolites, the Ingenuity Pathway Analysis evaluates the overrepresentation of metabolic pathways and mapped out the important pathways that are suspected to be impacted by the phenotypes of type 2 diabetes. Table 2 lists the top 5 canonical pathways the Ingenuity Pathway Analysis determined by using the identified metabolites. Among all the pathways, Adenine and Adenosine Salvage VI ( $p=7.53 \mathrm{E}-04)$ and Tyrosine Biosynthesis IV $(p=7.53 \mathrm{E}-04)$ have an overlap percentage of $50 \%$, indicating half of the metabolites in the pathway are significantly altered, and therefore these two pathways be impacted greatly by the phenotype of type 2 diabetes. Adenosine Nucleotides Degradation II $(\mathrm{p}=2.06 \mathrm{E}-04)$ and Purine Nucleotides Degradation II (Aerobic) ( $p=$ $8.13 \mathrm{E}-04)$ are as well obviously impacted, with an overlap percentage approximate to $30 \%(27.3 \%)$, and a 
percentage roughly equal to $18 \%(17.6 \%)$. In the pathway tRNA Charging $(\mathrm{p}=1.20 \mathrm{E}-03)$, only 4 of 43
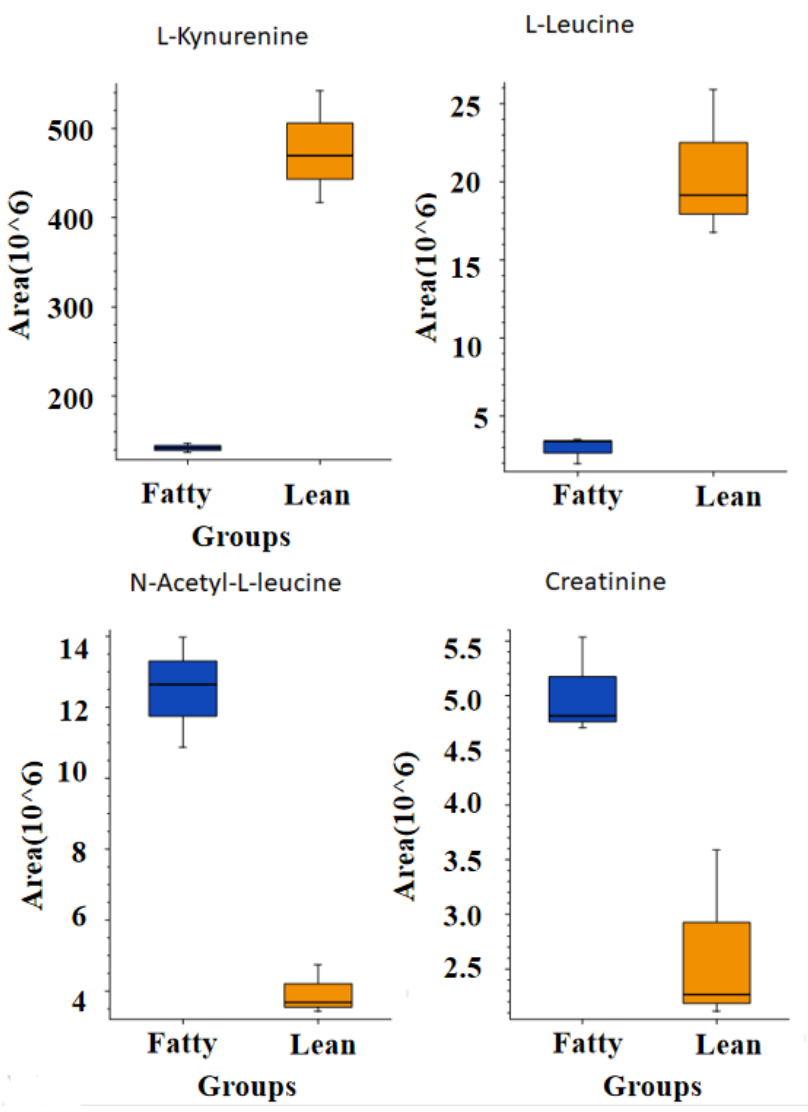

metabolites are considered significantly-altered, revealing a slighter impact of the phenotype.
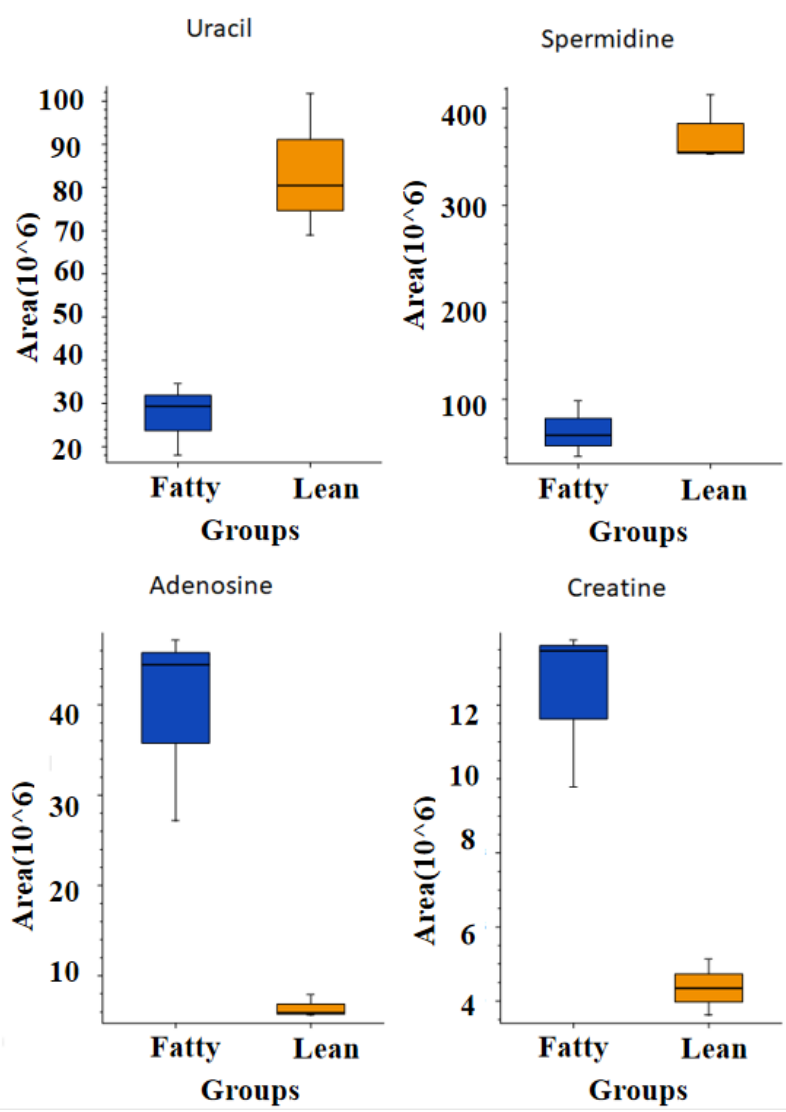

Fig. 3. Some of the significantly changed metabolites identified by MS/MS spectrum matching.

Table 2. P-value and the overlap percentage of the top 5 important pathways determined by the Ingenuity Pathway Analysis.

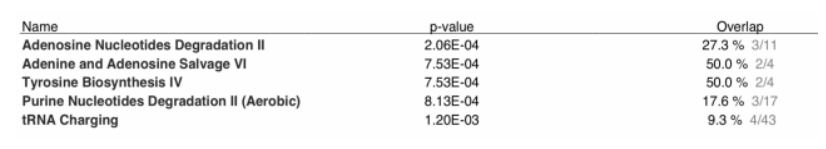

\subsection{Dysregulated pathways found through mummichog pathway analysis}

The mummichog algorithm uses metabolite sets as the unit of analysis and deduces altered metabolic pathways using the $\mathrm{m} / \mathrm{z}$ values from the MS peak results from untargeted metabolomics. A summary plot of all the predicted altered metabolic pathways is shown in figure 4. The significantly dysregulated pathways are listed in table 3. The mummichog algorithm suspected Tryptophan metabolism pathway $(\mathrm{p}=0.0013401)$ and Tyrosine metabolism pathway $(\mathrm{p}=0.01838)$ to be significantly impacted. There are 34 of 41 significantly altered spectral features in the Tryptophan metabolism pathway, resulting in a coverage percentage of approximately $82.9 \%$. Tyrosine metabolism pathway includes 34 of 42 spectral features significantly altered, resulting in a coverage percentage close to $81.0 \%$.

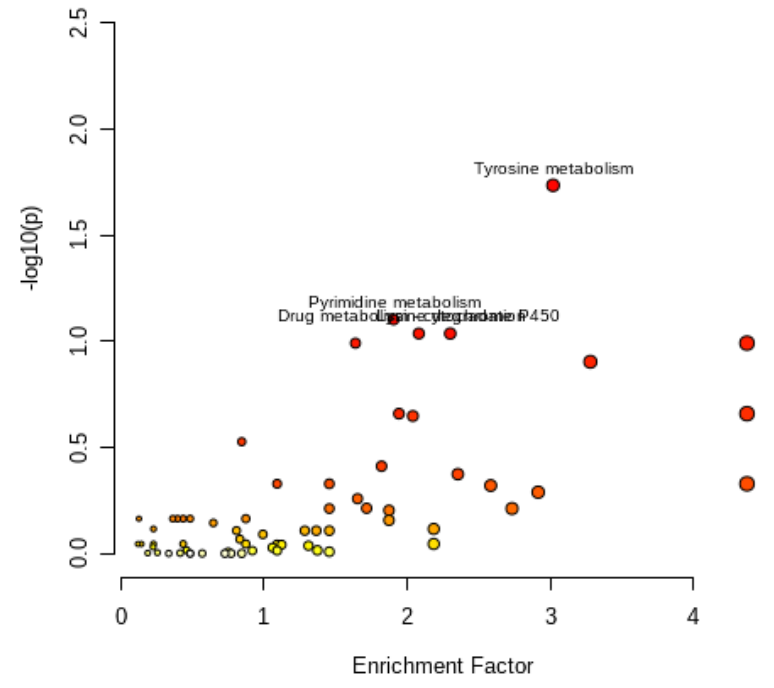

Fig. 4. P-value and significant features enrichment level of all the metabolic pathways estimated through mummichog pathway analysis. 
Table 3. Impacted metabolic pathways determined through mummichog analysis using $\mathrm{m} / \mathrm{z}$ values of the spectral features.

\begin{tabular}{|c|c|c|c|c|}
\hline Pathway Name & $\begin{array}{l}\text { Total Compounds } \\
\text { in the Pathway }\end{array}$ & Total Hits & $\begin{array}{c}\text { Expected } \\
\text { Hits }\end{array}$ & P-value \\
\hline Tryptophan metabolism & 41 & 34 & 9.3805 & 0.0013401 \\
\hline Tyrosine metabolism & 42 & 34 & 9.6093 & 0.01838 \\
\hline
\end{tabular}

This investigation analyzed the serum metabolites of Zucker Diabetic Fatty rats to study the underlying metabolic process and the pathology of type 2 diabetes. Through the Ingenuity Pathway Analysis and the Mummichog Pathway Analysis, we identified seven important pathways that were impacted by the phenotype of type 2 diabetes, Adenosine Nucleotides Degradation II, Adenine and Adenosine Salvage VI, Tyrosine Biosynthesis IV, Purine Nucleotides Degradation II (Aerobic), tRNA Charging, Tryptophan metabolism, and Tyrosine metabolism. They all play some important roles in the biological processes.

In many organisms, adenosine nucleotides degradation II degrades the nucleotide form (AMP) to urate and downwards, generating carbon, nitrogen, and energy [13,14]. The key enzyme in this pathway, adenosine deaminase, is widely classified in human tissues. It has a great impact in the reproduction and differentiation of lymphoid cells, and seems to impact different stages of the maturation of the immune system $[15,16]$. In addition to this, to generate energy continuously, many organisms recycle adenosine nucleotides by repeating the process of adenosine nucleotides degradation II and the adenine and adenosine salvage VI. Adenine and adenosine salvage VI is a pathway that converts adenosine back to AMP and the predecessor of superpathway of purine nucleotide de novo biosynthesis II that produces the indispensable compound for bioenergetic processes, nucleic acid synthesis and other courses of cell metabolism [17]. As it happens, a pathway predicted by the Ingenuity pathway analysis, purine nucleotides degradation II (aerobic), is the superpathway of adenosine nucleotides degradation II that has a great impact in nitrogen metabolism. Some organisms regarded purines as the only source of carbon and nitrogen while others use them to deposit and translocate nitrogen for assimilation and excretion [18]. The next pathway identified, Tyrosine Biosynthesis IV pathway, also called L-tyrosine Biosynthesis IV, converts L-phenylalanine into L-tyrosine and concentrates in the liver and the kidney [19,20,21]. The L-phenylalanine is an essential amino acid in human bodies that plays an important role in protein synthesis and the conversion of an indispensable protein component, L-tyrosine [21,22]. The last pathway predicted by the Ingenuity Pathway Analysis, tRNA charging attaches amino acids to tRNA in order for the further incorporation with the polypeptide. The tRNA is the carrier of amino acids and is known to translate genes into proteins [23]. It also acts as signaling molecules in the regulation of the metabolic and cellular processes of organisms, and a potent scavenger of cytochrome c [24].

The two pathways identified by the mummichog algorithm are general designations of chemicals reactions and pathways involving Tryptophan and
Tyrosine. Tryptophan is an essential amino acid that has a relatively low amount in humans [25]. It impacts mood, behavior, and the modulation of growth, feed intake, and immune responses [25]. Tyrosine, on the other hand, is also an amino acid and acts as a component of human proteins [26]. A number of steps are involved in the conversion of it into urea, carbon dioxide, and water and the formations of melanin, norepinephrine, epinephrine, thyroxine, and other important substances are impacted through this process [26]. Together, the Ingenuity analysis and the mummichog algorithm predicted seven pathways that have an important impact in organs, body systems, and the metabolic and cellular processes of humans, thus providing some insights into the discovery of therapeutic targets and the study in the pathology of type 2 diabetes.

\section{Conclusion}

Eight significantly changed metabolites in ZDF rats were identified. They were L-Kynurenine, L-Leucine, Uracil, Spermidine, N-Acetyl-L-leucine, Creatinine, Adenosine and Creatine. L-Kynurenine is a moderately acidic compound and has been found in most human tissues. It is also involved in the tryptophan metabolism pathway (PubChem, n.d.-b). L-Leucine is an essential amino acid in humans for protein synthesis and metabolic functions. It is involved in the 3-methylglutaconic aciduria type IV pathway, the 3-methylglutaconic aciduria type I pathway, and isovaleric acidemia pathway (PubChem, n.d.-a). Uracil belongs to organic compounds known as pyrimidines that contain pyrimidine rings which bears ketone. It has been detected in several foods, hence Uracil has potential as a biomarker. Spermidine is an aliphatic polyamine and it is in a variety of foods such as mushrooms, soy products and whole grains. N-Acetyl-Lleucine is known as leucine and derivatives (PubChem, n.d.-c)

We found seven potentially impacted pathways: adenosine Nucleotides Degradation II, Adenine and Adenosine Salvage VI, Tyrosine Biosynthesis IV, Purino Nucleotides Degradation II (Aerobic) and tRNA Charging, Tryptophan metabolism pathway and Tyrosine metabolism pathway. Tyrosine metabolic pathways. Identifying those metabolites and pathways is the first step in drug development. Now studying the result would help in discovery of therapeutic targets and the development of diabetes biomarkers.

\section{References}

Here are some examples:

1. Tanaka, Y.; Gleason, C. E.; Tran, P. O. T.; Harmon, J. S.; Robertson, R. P. Prevention of Glucose Toxicity in HIT-T15 Cells and Zucker Diabetic Fatty Rats by Antioxidants. Proc Natl Acad Sci USA 1999, 6.

2. Chatham, J. C.; Seymour, A.-M. L. Cardiac Carbohydrate Metabolism in Zucker Diabetic Fatty Rats. Cardiovasc. Res. 2002, 55 (1), 104-112. 
3. Klein, M. S.; Shearer, J. Metabolomics and Type 2 Diabetes: Translating Basic Research into Clinical Application. J. Diabetes Res. 11.

4. Henry, C. S. High-Throughput Generation, Optimization and Analysis of Genome-Scale Metabolic Models. Nat. Biotechnol. 2010, 28 (9), 8.

5. Szabadi, K.; Pinter, E.; Reglodi, D.; Gabriel, R. Neuropeptides, Trophic Factors, and Other Substances Providing Morphofunctional and Metabolic Protection in Experimental Models of Diabetic Retinopathy. 121.

6. Yokoi, N.; Hoshino, M.; Hidaka, S.; Yoshida, E.; Beppu, M.; Hoshikawa, R.; Sudo, K.; Kawada, A.; Takagi, S.; Seino, S. A Novel Rat Model of Type 2 Diabetes: The Zucker Fatty Diabetes Mellitus ZFDM Rat. J. Diabetes Res. 2013, 2013, 1-9. https://doi.org/10.1155/2013/103731.

7. Lewis, N. E.; Nagarajan, H.; Palsson, B. O. Constraining the Metabolic Genotype-Phenotype Relationship Using a Phylogeny of in Silico Methods. Nat. Rev. Microbiol. 2012, 10 (4), 291305. https://doi.org/10.1038/nrmicro2737.

8. Zhou, B.; Xiao, J. F.; Tuli, L.; Ressom, H. W. LCMS-Based Metabolomics. Mol BioSyst 2012, 8 (2), 470-481. https://doi.org/10.1039/C1MB05350G.

9. Podwojski, K.; Fritsch, A.; Chamrad, D. C.; Paul, W.; Sitek, B.; Stühler, K.; Mutzel, P.; Stephan, C.; Meyer, H. E.; Urfer, W.; Ickstadt, K.; Rahnenführer, J. Retention Time Alignment Algorithms for LC/MS Data Must Consider Non-Linear Shifts. 7.

10. Friedrich, N. Metabolomics in Diabetes Research. 33.

11. Coughlan, K. A.; Ruderman, N. B.; Valentine, R. J.; Saha, A. K. AMPK Activation: A Therapeutic Target for Type 2 Diabetes? 13.

12. Chou, K.-C. Molecular Therapeutic Target for Type-2 Diabetes. J. Proteome Res. 2004, 3 (6), 1284-1288. https://doi.org/10.1021/pr049849v.

13. Kahn, K.; Serfozo, P.; Tipton, P. A. Identification of the True Product of the Urate Oxidase Reaction. J. Am. Chem. Soc. 1997, 119 (23), 5435-5442. https://doi.org/10.1021/ja970375t.

14. Xi, H.; Schneider, B. L.; Reitzer, L. Purine Catabolism in Escherichia Coli and Function of Xanthine Dehydrogenase in Purine Salvage. J. Bacteriol. 2000, 182 (19), 5332-5341. https://doi.org/10.1128/JB.182.19.5332-5341.2000.

15. Elshafei, A. M.; Mohamed, L. A.; Ali, N. H. Deamination of Adenosine by Extracts of Penicillium Politans NRC-510. J. Basic Microbiol. 2005, $45 \quad$ (2), $115-124$. https://doi.org/10.1002/jobm.200410476.

16. Yoneyama, Y.; Sawa, R.; Suzuki, S.; Otsubo, Y.; Araki, T. Serum Adenosine Deaminase Activity in Women with Hyperemesis Gravidarum. Clin. Chim. Acta 2002, 5.

17. Ashihara, H.; Stasolla, C.; Loukanina, N.; Thorpe, T. A. Purine Metabolism during White Spruce Somatic
Embryo Development: Salvage of Adenine, Adenosine, and Inosine. Plant Sci. 2001, 11.

18. Pope, S. D.; Chen, L.-L.; Stewart, V. Purine Utilization by Klebsiella Oxytoca M5al: Genes for Ring-Oxidizing and -Opening Enzymes. J. Bacteriol. 2009, $191 \quad$ (3), $1006-1017$. https://doi.org/10.1128/JB.01281-08.

19. McGee, M. M.; Greengard, O.; Knox, W. E. Liver Phenylalanine Hydroxylase Activity in Relation to Blood Concentrations of Tyrosine and Phenylalanine in the Rat. Biochem. J. 1972, 127 (4), 675-680. https://doi.org/10.1042/bj1270675.

20. Stanley, J. C.; Fisher, M. J.; POGSONt, C. I. The Metabolism of L-Phenylalanine and L-Tyrosine by Liver Cells Isolated from Adrenalectomized Rats and from Streptozotocin-Diabetic Rats. 1985, 228, 7.

21. Moller, N.; Meek, S.; Bigelow, M.; Andrews, J.; Nair, K. S. The Kidney Is an Important Site for in Vivo Phenylalanine-to-Tyrosine Conversion in Adult Humans: A Metabolic Role of the Kidney. Proc. Natl. Acad. Sci. 2000, 97 (3), 1242-1246. https://doi.org/10.1073/pnas.97.3.1242.

22. Womack, M.; Rose, W. C. Feeding Experiments with Mixtures of Highly Purified Amino Acids. 6. The Relation of Phenylalanine and Tyrosine to Growth. J. Biol. Chem. 1934, 107, 449-458.

23. Zhou, Y.; Goodenbour, J. M.; Godley, L. A.; Wickrema, A.; Pan, T. High Levels of TRNA Abundance and Alteration of TRNA Charging by Bortezomib in Multiple Myeloma. Biochem. Biophys. Res. Commun. 2009, 385 (2), 160-164. https://doi.org/10.1016/j.bbrc.2009.05.031.

24. Raina, M.; Ibba, M. TRNAs as Regulators of Biological Processes. Front. Genet. 14.

25. Floc'h, N. L.; Otten, W.; Merlot, E. Tryptophan Metabolism, from Nutrition to Potential Therapeutic Applications. 11.

26. Woolf, L. I. Inherited Metabolic Disorders: Errors of Phenylalanine and Tyrosine Metabolism. In Advances in Clinical Chemistry; Sobotka, H., Stewart, C. P., Eds.; Elsevier, 1963; Vol. 6, pp $97-$ 230. https://doi.org/10.1016/S0065-2423(08)602395. 